\title{
Recombinant thrombomodulin does not promote bacterial propagation but alleviates oxidative stress in rats infected with methicillin-resistant Staphylococcus aureus
}

Takashi Ito ( $\nabla$ takashi@m3.kufm.kagoshima-u.ac.jp )

Kagoshima University https://orcid.org/0000-0002-6204-8400

Binita Shrestha

University of Michigan

Yasuyuki Kakihana

Kagoshima University

Ikuro Maruyama

Kagoshima University

Research

Keywords: Sepsis, MRSA, immunothrombosis, thrombomodulin, metabolomics, oxidative stress

Posted Date: May 22nd, 2020

DOI: https://doi.org/10.21203/rs.3.rs-29565/v1

License: (c) (1) This work is licensed under a Creative Commons Attribution 4.0 International License.

Read Full License 


\section{Abstract \\ Background}

Recombinant thrombomodulin (rTM) has been used for treatment of sepsis-associated disseminated intravascular coagulation. Recent studies have suggested that anticoagulant therapy might dampen the protective role of immunothrombosis. We examined if rTM might worsen infectious diseases.

\section{Methods}

Male Sprague-Dawley rats with jugular-vein catheterization were divided into three groups: no anticoagulation; rTM pretreatment; rTM treatment at $6 \mathrm{~h}$. Live methicillin-resistant Staphylococcus aureus (MRSA) was inoculated into the tail vein of rats. rTM was administered into the jugular-vein catheter before or $6 \mathrm{~h}$ after MRSA inoculation, while an equal volume of saline was administered in the noanticoagulation group. Blood samples were collected from the jugular-vein catheter before, $6 \mathrm{~h}$ and $12 \mathrm{~h}$ after MRSA inoculation. Tissue samples were collected from anesthetized rats when moribund or $18 \mathrm{~h}$ after MRSA inoculation.

\section{Results}

The survival rate of rats in the no-anticoagulation group, rTM pretreatment group, and rTM treatment at 6h group was $50 \%, 25 \%$, and $75 \%$, respectively. Bacterial burden in blood, lung, liver, and spleen was neither increased nor decreased in rats treated with $\mathrm{rTM}$. The ratio of bacteria found in the extravascular space to those in the intravascular space was increased in rats treated with rTM. Metabolomics analysis revealed that rTM treatment alleviated oxidative stress, as evidenced by the decrease in levels of oxidized glutathione.

\section{Conclusions}

rTM does not promote bacterial propagation but alleviates oxidative stress in a rat model of bloodstream infection with MRSA.

\section{Background}

Sepsis can be defined as life-threatening organ dysfunction caused by dysregulated host responses to infection (1). Dysregulated host responses include feedforward neutrophil accumulation, overproduction of reactive oxygen species, release of neutrophil extracellular traps (NETs), and a metabolic shift towards decreased oxidation of fatty acids (2-4). Intravascular activation of coagulation is another important feature of the host response to infection $(2,5)$. Expression of tissue factor on monocytes and release of NETs from neutrophils are thought to be triggers for intravascular coagulation $(5,6)$. Coagulation under 
certain circumstances has a major physiologic role in innate immune defense against infection, a process known as "immunothrombosis". However, uncontrolled immunothrombosis may lead to ischemic organ failure.

Several mechanisms have been proposed by which immunothrombosis promotes defense against infection (6). First, immunothrombosis limits microbial dissemination by arresting microbes within the clot (7). Second, thrombi form "barricades" which can limit microbial spread from one compartment to another $(8,9)$. Third, fibrin supports leukocytes in eliminating microbes (10). Consequently, mice pretreated with coumadin have been shown to display increased hepatic bacterial burden and mortality after inoculation with Yersinia enterocolitica (11).

Anticoagulant therapy has been considered as a treatment option for sepsis. It is not efficacious in sepsis overall, but may provide beneficial effects in a specific subpopulation with sepsis-associated disseminated intravascular coagulation (DIC) $(12,13)$, possibly by alleviating ischemic organ failure. Among anticoagulants, recombinant thrombomodulin (rTM) is a potential therapeutic agent for sepsisassociated DIC (14). Meta-analyses have suggested a potential survival benefit using this drug $(15,16)$, and rTM is now used widely in Japan. However, one might assume that treatment with rTM "dampens" the protective role of immunothrombosis and, thus, may worsen infectious diseases. To validate the plausibility of this hypothesis, we examined bacterial burden in organs and blood in the presence or absence of rTM in methicillin-resistant Staphylococcus aureus (MRSA)-infected rats. We also examined if the timing of rTM administration, before or after MRSA inoculation, affected bacterial burden.

\section{Methods}

\section{Model of MRSA infection in rats}

Experiments involving animals were approved by the Animal Care and Use Committee of Kagoshima University and Shin Nippon Biomedical Laboratories.

Male Sprague-Dawley rats with jugular-vein catheterization were used for all experiments. MRSA (ATCC43300) was incubated with agitation in Mueller-Hinton broth (Eiken Chemicals, Tokyo, Japan) for $2.5 \mathrm{~h}$ at $37^{\circ} \mathrm{C}$ to achieve mid-log-phase growth. Then, MRSA was resuspended in saline, and $3.5 \times 10^{8}$ colonyforming units (CFUs) of MRSA was inoculated into the tail vein of rats at $0 \mathrm{~h}$ and $5 \mathrm{~h}$. Anticoagulation therapy was conducted in some rats, for which rTM ( $1 \mathrm{mg} / \mathrm{kg}$; Asahi Kasei Pharma, Tokyo, Japan) was administered into the jugular-vein catheter immediately before (rTM pretreatment group) or $6 \mathrm{~h}$ after the first MRSA inoculation (rTM treatment at 6-h group). The general condition of rats was evaluated every $1 \mathrm{~h}$. Rats were sacrificed when moribund or $18 \mathrm{~h}$ after the first MRSA inoculation ( $\mathrm{n}=8$ in each group).

\section{Blood tests}

Blood samples were collected from the jugular-vein catheter before, $6 \mathrm{~h}$ and $12 \mathrm{~h}$ after MRSA inoculation ( $n=5$ in each group). One portion of blood samples was used for bacteriology. Blood samples 
anticoagulated with ethylenediaminetetraacetic acid were used for automated counting of blood cells by an ADVIA120 system (Bayer Diagnostics, Leverkusen, Germany). Serum samples were used for measurement of levels of total bilirubin and creatinine by a clinical biochemistry analyzer (BioMajesty ${ }^{\text {TM }}$ JCA-BM6070; Jeol, Tokyo, Japan). Citrated plasma samples were used for metabolomics analysis.

\section{Bacteriology and pathology}

Anesthetized rats were sacrificed when moribund or $18 \mathrm{~h}$ after MRSA inoculation. Small samples of liver, spleen, kidney, and lung were homogenized in sterile phosphate-buffered saline (PBS, $1 \mathrm{~mL} / \mathrm{g}$ tissue), and used for bacteriology ( $n=5$ in each group). The remainder of the organs were fixed in $10 \%$ neutralbuffered formalin, and used for pathology studies ( $n=3$ in each group). Samples of blood and tissue homogenates were serially diluted with sterile PBS, plated onto sheep blood agar plates (Nissui Pharmaceuticals, Tokyo, Japan), and incubated overnight at $37^{\circ} \mathrm{C}$ before bacterial enumeration. Bacterial distribution in each organ was analyzed using Gram-stained tissue sections.

\section{Metabolomics analysis}

Metabolomics analysis was undertaken at a service facility of LSI Medience (Tokyo, Japan). Briefly, plasma samples $(200 \mu \mathrm{L})$ collected before and $12 \mathrm{~h}$ after MRSA inoculation were mixed with methanol $(800 \mu \mathrm{L})$ and shaken for $15 \mathrm{~min}$ at room temperature. After centrifugation, the precipitates were dissolved with $10 \%$ acetonitrile aqueous solution $(200 \mu \mathrm{L})$. After addition of internal standards, they were analyzed by liquid chromatography-mass spectrometry (LC-MS) and capillary electrophoresis-mass spectrometry (CE-MS). Tuning and calibration were carried out with standard solutions provided by Agilent Technologies (Palo Alto, CA, USA).

LC-MS was done using a LC system (HP1200; Agilent Technologies) equipped with a C18 column ( $2 \mu \mathrm{m}$, $50 \mathrm{~mm} \times 2.0 \mathrm{~mm}$ ID, CAPCELL PAK C18 IF; Shiseido, Tokyo, Japan) coupled with an electrospray ionization quadrupole time-of-flight mass spectrometer (6520; Agilent Technologies). Solvent A was composed of $5 \mathrm{mM}$ ammonium acetate aqueous solution, whereas solvent $\mathrm{B}$ was acetonitrile.

Metabolites were eluted at a flow rate of $0.2 \mathrm{~mL} / \mathrm{min}$ at $40{ }^{\circ} \mathrm{C}$ with a linear gradient of $10-90 \%$ solvent $\mathrm{B}$ over $10 \mathrm{~min}$, followed by a further 5 -min hold at $100 \%$ solvent $B$. The mass spectrometer was operated in positive and negative scan modes $(\mathrm{m} / z 60$ to 1,200$)$ with a capillary voltage of $3,500 \mathrm{~V}$. The nebulizing gas pressure was 40 psi and the dry gas flow was $8 \mathrm{~L} / \mathrm{min}$ at $350^{\circ} \mathrm{C}$.

Ionic metabolites were measured in the positive mode of a CE-time-of-flight mass spectrometer (6520; Agilent Technologies). Metabolites were separated in a fused-silica capillary (50 $\mu \mathrm{m}$ i.d. $\times 100 \mathrm{~cm}$ total length; GL Science, Tokyo, Japan) filled with $1 \mathrm{~mol} / \mathrm{L}$ of formic acid aqueous solution (cation mode), or $20 \mathrm{mM}$ ammonium formate and $20 \mathrm{mM}$ ammonium acetate aqueous solution ( $\mathrm{pH} \mathrm{10}$, anion mode) as the electrolyte. The sample solution was injected at $5 \mathrm{kPa}$ for $15 \mathrm{~s}(\sim 15 \mathrm{~nL})$, and a voltage of $30 \mathrm{kV}$ was applied. The capillary tray and sample tray were maintained at room temperature and $5{ }^{\circ} \mathrm{C}$, respectively. The sheath liquid was methanol/water $(50 \% \mathrm{v} / \mathrm{v})$ containing $5 \mathrm{mM}$ ammonium acetate. The CE-time of flight mass spectrometer was operated in positive and negative scan modes $(\mathrm{m} / z 60$ to 1,200$)$. The 
capillary voltage was set at $3,500 \mathrm{~V}$ and the nitrogen gas (heater temperature $=250{ }^{\circ} \mathrm{C}$ ) flow rate was set at $10 \mathrm{~L} / \mathrm{min}$.

A data file of MS was converted to csv format with csv convertor (Agilent Technologies). All peak positions (retention time and $\mathrm{m} / \mathrm{z}$ ) and areas were calculated by Markeranalysis (LSI Medience). All peak areas were aligned into one datasheet and the errors of peak intensities were corrected by internal standards. Noise peaks were deleted compared with the peaks detected in blank samples. Metabolites were identified by comparing the retention time and $\mathrm{m} / \mathrm{z}$ with the standard data set established by LSI Medience. Mean fold-change and the Student's $t$-test were carried out for all detected peaks.

\section{Statistical analyses}

Statistical analyses were carried out using SPSS v26 (IBM. Armonk NY, USA). For comparison of independent groups, the Student's t-test was used. $P<0.05$ was considered significant.

\section{Results}

To test whether anticoagulation with rTM could worsen infectious diseases, we first analyzed the survival rates of MRSA-infected rats in the presence or absence of rTM administration. The survival rate of rats without rTM administration was $50 \%$ at the study endpoint. The survival rate of rats treated with rTM immediately before or $6 \mathrm{~h}$ after MRSA inoculation was 25\% and 75\%, respectively. Hence, the timing of rTM administration might be an important determinant of treatment efficacy.

Next, we conducted hematology and bacteriology studies to ascertain the effects of rTM administration on MRSA-induced disease. Leukopenia became evident $6 \mathrm{~h}$ after MRSA inoculation regardless of treatment (Fig. 2B). Thrombocytopenia was less prominent in rats treated with rTM when compared with those without anticoagulation, though the difference was not significant (Fig. 2C). Liver dysfunction and kidney dysfunction, as evidenced by an increase in the level of total bilirubin and creatinine, respectively, became prominent $12 \mathrm{~h}$ after MRSA inoculation regardless of treatment (Fig. 2D, E). The bacterial burden in samples of blood, lung, liver, and spleen was neither increased nor decreased in rats treated with rTM (Fig. 3A, B). However, the ratio of bacteria found in the extravascular space to those found in the intravascular space was increased in rats treated with rTM (Fig. 3C, D). These findings suggested that rTM treatment could allow bacterial passage from the intravascular space to the extravascular space, but bacterial burden in the whole organ may be only slightly affected by rTM treatment.

Next, we analyzed metabolites in the plasma of MRSA-infected rats comprehensively to ascertain the potential effects of rTM administration. A total of 209 metabolites of known structures were identified and profiled. The relative abundance and $P$-value of each metabolite in the rTM treatment at 6-h group with reference to the no-anticoagulation group or rTM pretreatment group are shown in Fig. 4A, and the identity of each metabolite is shown in Supplementary Table. Among the metabolites, levels of oxidized glutathione, glycodeoxycholate, and uridine 5'-diphosphate were decreased significantly in the rTM treatment at 6-h group compared with those in the no-anticoagulation group, whereas octanoylcarnitine 
levels were increased significantly (Fig. 4B). These findings suggested that rTM treatment may alleviate oxidative stress, bile-acid stasis, nucleotide accumulation, and recover fatty-acid metabolism.

\section{Discussion}

Recent studies have raised fears that anticoagulant therapy might dampen the protective role of immunothrombosis and, thus, might worsen infectious diseases. Using a rat model of bloodstream infection with MRSA, we found that treatment with rTM neither increased bacterial burden nor worsened overall survival if rTM was used after the infection. Instead, rTM treatment alleviated oxidative stress, as evidenced by the decrease in the level of oxidized glutathione.

Bacterial passage from one compartment to another (e.g., from the intravascular space to the extravascular space) was facilitated by rTM treatment in our study. This finding might not be specific to rTM but common to various anticoagulants, including hirudin (8) and heparin (data not shown). Thus, we cannot completely deny the potential risk of bacterial dissemination by anticoagulant therapy. However, the increase of bacteria in the extravascular space seemed to be accompanied by a decrease of bacteria in the intravascular space, and so the bacterial burden in the whole organ was little-affected by rTM treatment. Considering that rTM did not worsen the survival rate of MRSA-infected rats if used after the infection, rTM treatment might not dampen the protective role of immunothrombosis.

Metabolomics analysis revealed that the increase in the level of oxidized glutathione in response to MRSA infection was alleviated by rTM administration $6 \mathrm{~h}$ after MRSA infection. Glutathione plays a major part in cellular defenses against oxidative stress (17). In exchange for the reduction of target proteins, glutathione (also known as reduced glutathione or GSH) is converted to glutathione disulfide (also known as oxidized glutathione or GSSG). The GSSG:GSH ratio is thought to be a marker of cellular toxicity and is associated with mortality in septic-shock patients (18). In the present study, the GSSG:GSH ratio was also increased in response to MRSA infection, and was decreased by rTM administration $6 \mathrm{~h}$ after MRSA infection (data not shown). These findings suggest that rTM alleviates oxidative stress, possibly through the maintenance of vascular patency or its intrinsic antioxidant properties (19).

The timing of anticoagulation might also be controversial. Anticoagulant drugs have been administered before infection in studies for immunothrombosis $(8,11)$ whereas rTM has been administered after infection onset in clinical settings (20). We administered rTM immediately before or $6 \mathrm{~h}$ after MRSA infection, and found that rTM administration $6 \mathrm{~h}$ after MRSA infection might be better in terms of improved survival. Although the best timing for clinical anticoagulation was not specified in our study, signs of organ dysfunction in addition to evident DIC might be important. Further studies are needed to gain insight into the appropriate timing for anticoagulation during sepsis treatment.

Our study had several limitations. First, it is not clear whether our findings in rats can be transferred to humans. Although studies have shown that rTM is effective in mitigating DIC in rats and humans (20, 21), activated protein C-dependent actions might not be fully expected in rats, unlike humans (22). Second, it is not clear whether our findings are applicable to infection models other than bloodstream 
infection with MRSA. Third, observation beyond $18 \mathrm{~h}$ was not possible in this study because rats became seriously ill at about this point. Fourth, the number of rats in each group was low due in part to the complexity of experiments using rats with jugular-vein catheterization and live bacteria. Fifth, erroneous inferences may have occurred when multiple comparisons were conducted simultaneously in metabolomics analysis. Further studies are necessary to confirm the findings of our study.

\section{Conclusions}

rTM does not promote bacterial propagation but alleviated oxidative stress in a rat model of bloodstream infection with MRSA.

\section{Abbreviations}

CFU

colony-forming unit

DIC

disseminated intravascular coagulation

GSH

reduced glutathione

GSSG

oxidized glutathione

MRSA

methicillin-resistant Staphylococcus aureu

NETs

neutrophil extracellular traps

PBS

phosphate buffered saline

rTM

recombinant thrombomodulin

UDP

uridine $5^{\prime}$-diphospate

\section{Declarations}

\section{Ethics approval and consent to participate}

Experiments involving animals were approved by the Animal Care and Use Committee of Kagoshima University and Shin Nippon Biomedical Laboratories, which are both in Kagoshima, Japan.

\section{Consent for publication}


Not applicable.

\section{Availability of data and material}

The datasets used and/or analyzed during the current study are available from the corresponding author on reasonable request.

\section{Competing interests}

$\mathrm{TI}$ and IM received research grants from Asahi Kasei Pharma, a pharmaceutical company manufacturing recombinant thrombomodulin. The other authors have no conflicts of interest.

\section{Funding}

This work was supported by research grants from the Japan Society for the Promotion of Science (Grants-in-Aid 15H05684 and 18K16543).

\section{Authors' contributions}

TI designed the experimental protocol and wrote the manuscript. BS participated in the laboratory experiments. IM and YK critically appraised the manuscript. All authors read and approved the final manuscript.

\section{Acknowledgements}

The authors thank Yasuharu Otsubo (Shin Nippon Biomedical Laboratories), Kazuhiro Tanabe (LSI Medience Corporation), and Ryo Sugimoto (Kagoshima University) for their excellent technical assistance. We thank Arshad Makhdum, PhD, from Edanz Group (www.edanzediting.com/ac) for editing a draft of this manuscript.

\section{References}

1. Singer M, Deutschman CS, Seymour CW et al (2016) The Third International Consensus Definitions for Sepsis and Septic Shock (Sepsis-3). JAMA 315(8):801-810

2. van der Poll T, van de Veerdonk FL, Scicluna BP et al (2017) The immunopathology of sepsis and potential therapeutic targets. Nat Rev Immunol 17(7):407-420

3. Brandes M, Klauschen F, Kuchen S et al (2013) A systems analysis identifies a feedforward inflammatory circuit leading to lethal influenza infection. Cell 154(1):197-212 
4. Cheng SC, Scicluna BP, Arts RJ et al (2016) Broad defects in the energy metabolism of leukocytes underlie immunoparalysis in sepsis. Nat Immunol 17(4):406-413

5. Ito T (2014) PAMPs and DAMPs as triggers for DIC. Journal of Intensive Care 2(1):67

6. Engelmann B, Massberg S (2013) Thrombosis as an intravascular effector of innate immunity. Nat Rev Immunol 13(1):34-45

7. Loof TG, Morgelin M, Johansson L et al (2011) Coagulation, an ancestral serine protease cascade, exerts a novel function in early immune defense. Blood 118(9):2589-2598

8. Massberg S, Grahl L, von Bruehl ML et al (2010) Reciprocal coupling of coagulation and innate immunity via neutrophil serine proteases. Nat Med 16(8):887-896

9. Macrae FL, Duval C, Papareddy P et al (2018) A fibrin biofilm covers blood clots and protects from microbial invasion. J Clin Investig 128(8):3356-3368

10. Flick MJ, Du X, Witte DP et al (2004) Leukocyte engagement of fibrin(ogen) via the integrin receptor alphaMbeta2/Mac-1 is critical for host inflammatory response in vivo. J Clin Investig 113(11):15961606

11. Luo D, Szaba FM, Kummer LW et al (2011) Protective roles for fibrin, tissue factor, plasminogen activator inhibitor-1, and thrombin activatable fibrinolysis inhibitor, but not factor $\mathrm{XI}$, during defense against the gram-negative bacterium Yersinia enterocolitica. J Immunol 187(4):1866-1876

12. Kienast J, Juers M, Wiedermann CJ et al (2006) Treatment effects of high-dose antithrombin without concomitant heparin in patients with severe sepsis with or without disseminated intravascular coagulation. J Thromb Haemost 4(1):90-97

13. Umemura $\mathrm{Y}$, Yamakawa $\mathrm{K}$, Ogura $\mathrm{H}$ et al (2016) Efficacy and safety of anticoagulant therapy in three specific populations with sepsis: a meta-analysis of randomized controlled trials. J Thromb Haemost 14(3):518-530

14. Ito $\mathrm{T}$, Thachil $\mathrm{J}$, Asakura $\mathrm{H}$ et al (2019) Thrombomodulin in disseminated intravascular coagulation and other critical conditions-a multi-faceted anticoagulant protein with therapeutic potential. Crit Care 23(1):280

15. Yamakawa K, Levy JH, Iba T (2019) Recombinant human soluble thrombomodulin in patients with sepsis-associated coagulopathy (SCARLET): an updated meta-analysis. Crit Care 23(1):302

16. Valeriani E, Squizzato A, Gallo A et al. Efficacy and safety of recombinant human soluble thrombomodulin in patients with sepsis-associated coagulopathy: a systematic review and metaanalysis. J Thromb Haemost 2020

17. Biolo G, Antonione R, De Cicco M (2007) Glutathione metabolism in sepsis. Crit Care Med 35(9 Suppl):S591-S595

18. Kim JS, Kwon WY, Suh GJ et al (2016) Plasma glutathione reductase activity and prognosis of septic shock. J Surg Res 200(1):298-307

19. Yamaji K, Wang Y, Liu Y et al (2005) Activated protein C, a natural anticoagulant protein, has antioxidant properties and inhibits lipid peroxidation and advanced glycation end products 
formation. Thromb Res 115(4):319-325

20. Hayakawa M, Yamakawa K, Saito S et al (2016) Recombinant human soluble thrombomodulin and mortality in sepsis-induced disseminated intravascular coagulation. A multicentre retrospective study. Thromb Haemost 115(6):1157-1166

21. Aoki Y, Ohishi R, Takei R et al (1994) Effects of recombinant human soluble thrombomodulin (rhsTM) on a rat model of disseminated intravascular coagulation with decreased levels of plasma antithrombin III. Thromb Haemost 71(4):452-455

22. Mohri M, Gonda Y, Oka M et al (1997) The antithrombotic effects of recombinant human soluble thrombomodulin (rhsTM) on tissue factor-induced disseminated intravascular coagulation in crabeating monkeys (Macaca fascicularis). Blood Coagul Fibrinolysis 8(5):274-283

\section{Figures}


Fig. 1

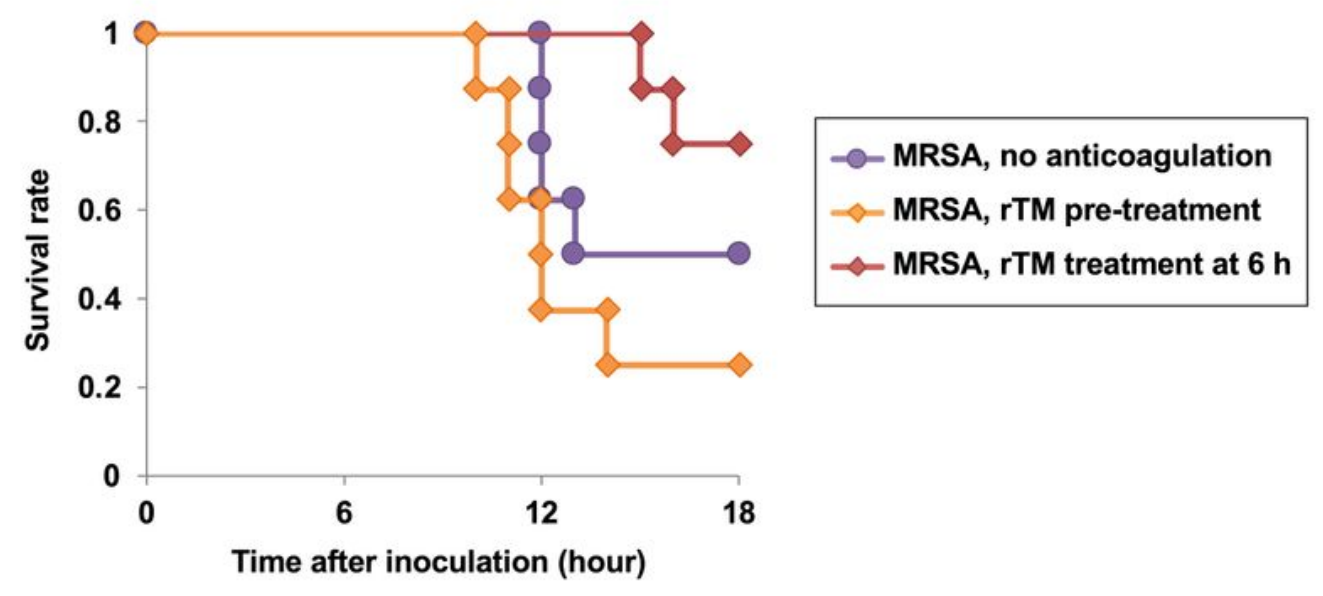

\section{Figure 1}

Survival benefit of treatment with recombinant thrombomodulin in MRSA-infected rats. MRSA $(3.5 \times 108$ $\mathrm{CFU})$ was inoculated into the tail vein of rats at $0 \mathrm{~h}$ and $5 \mathrm{~h}$. $\mathrm{rTM}(1 \mathrm{mg} / \mathrm{kg})$ was administered into a jugular-vein catheter immediately before (rTM pretreatment group) or $6 \mathrm{~h}$ after the first MRSA inoculation (rTM treatment at 6-h group) while an equal volume of saline was administered in the no-anticoagulation 
group. The general condition of rats was evaluated every $1 \mathrm{~h}$. Rats were sacrificed when moribund or $18 \mathrm{~h}$ after the first MRSA inoculation ( $\mathrm{n}=8$ in each group). Survival rates up to $18 \mathrm{~h}$ are shown.

Fig. 2
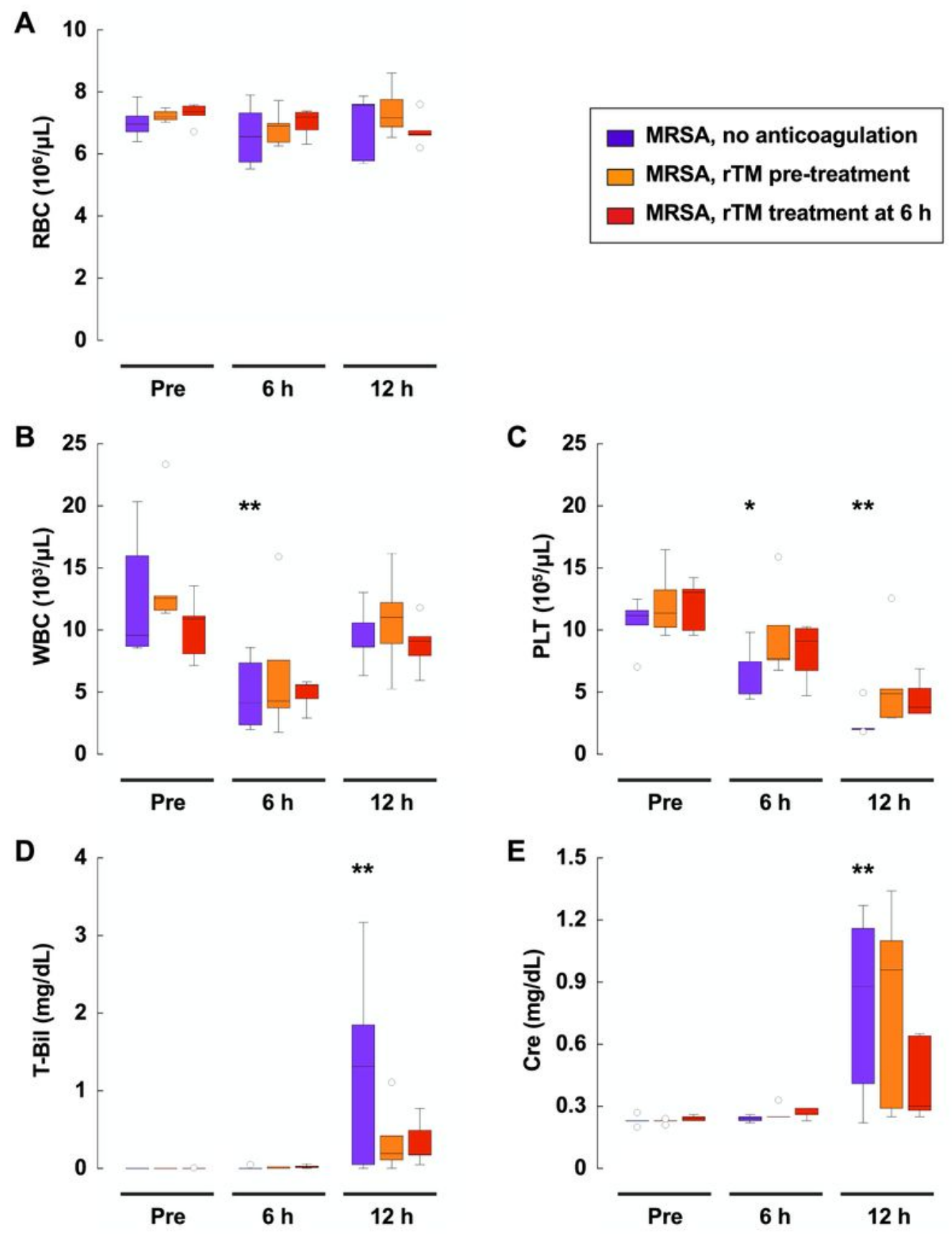

Figure 2

Thrombocytopenia and organ dysfunction in MRSA-infected rats. Blood samples were collected from a jugular-vein catheter before, $6 \mathrm{~h}$ and $12 \mathrm{~h}$ after MRSA inoculation ( $\mathrm{n}=5$ in each group). (A-C) Red blood cell (RBC), white blood cell (WBC), and platelet (PLT) counts in the no-anticoagulation group, rTM 
pretreatment group, and rTM treatment at 6-h group are shown. (D) Serum level of total bilirubin and (E) serum level of creatinine in the no-anticoagulation group, rTM pretreatment group, and rTM treatment at 6-h group are shown. Box plots show the median, lower and upper quartiles, lower and upper extreme values, and outliers. ${ }^{*} \mathrm{P}<0.05$ and ${ }^{* *} \mathrm{P}<0.01$ compared with data before MRSA inoculation.

Fig. 3
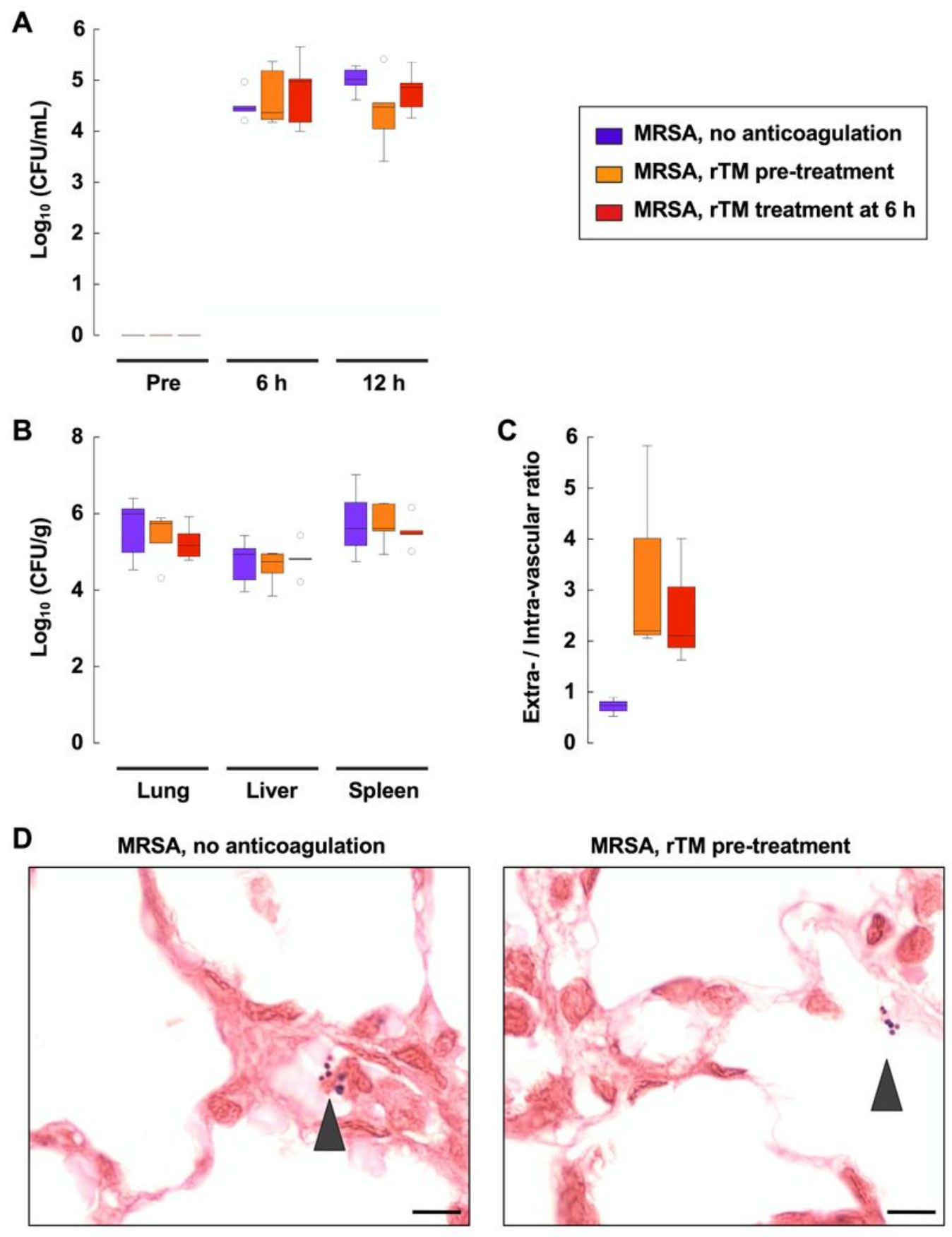

Figure 3 
Bacterial burden in MRSA-infected rats treated with recombinant thrombomodulin. (A) Blood samples collected before, $6 \mathrm{~h}$ and $12 \mathrm{~h}$ after MRSA inoculation were serially diluted, plated onto sheep blood agar plates, and incubated overnight at $37^{\circ} \mathrm{C}$ before bacterial enumeration. CFUs of blood samples in the noanticoagulation group, rTM pretreatment group, and rTM treatment at 6-h group are shown in a logarithmic scale ( $n=5$ in each group). (B) CFUs of tissue homogenates of lung, liver, and spleen are shown in a logarithmic scale ( $n=5$ in each group). (C) MRSA distribution in the lung was analyzed using Gram-stained tissue sections. The number of MRSA in the extravascular and intravascular space was counted separately, and the ratio is shown ( $n=3$ in each group). (D) Representative images of Gramstained lung tissue sections in the no-anticoagulation group and rTM pretreatment group are shown. Arrowheads show Gram-positive MRSA. Scale bar $=10 \mu \mathrm{m}$. 
Fig. 4

A
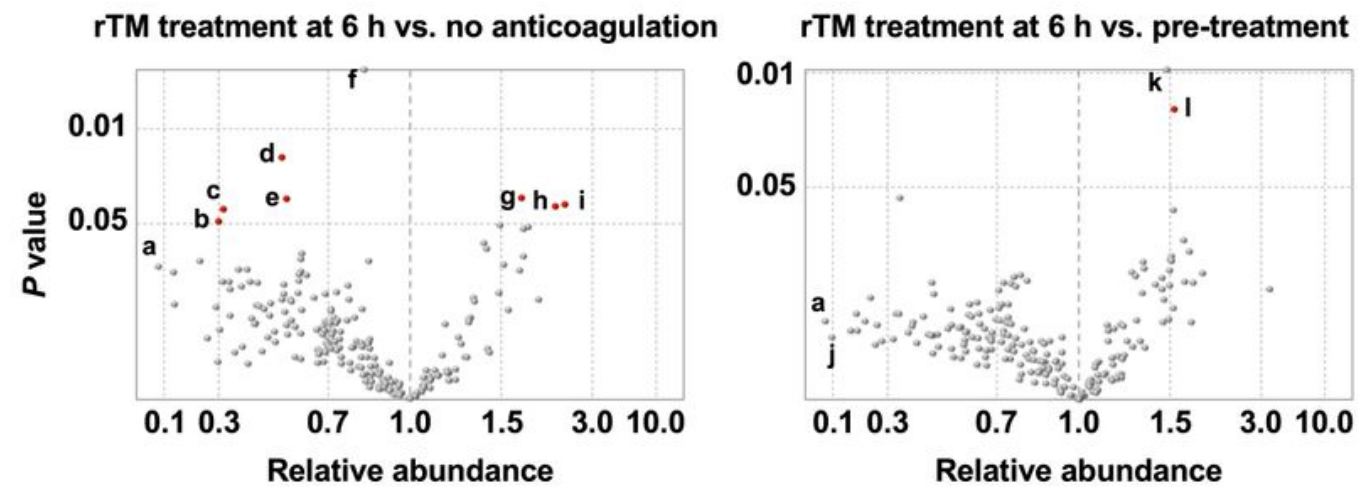

B
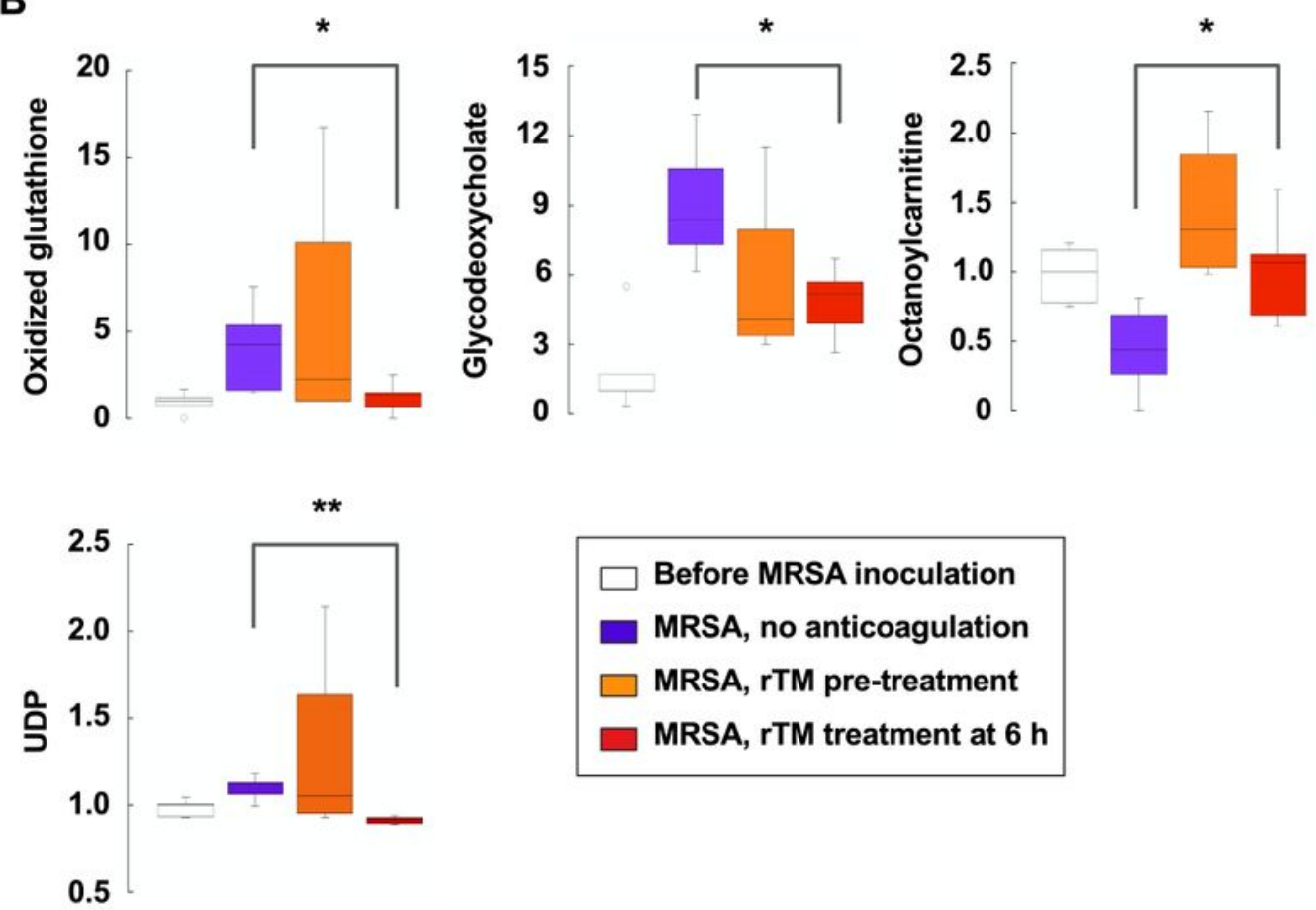

0.5

\section{Figure 4}

Metabolomics analysis suggests an antioxidative action of recombinant thrombomodulin in MRSAinfected rats. (A) Citrated plasma samples collected before and $12 \mathrm{~h}$ after MRSA inoculation were used for metabolomics analysis. Relative abundance and the P-value of each metabolite in the rTM treatment at 6-h group $(\mathrm{n}=5)$ with reference to the no-anticoagulation group $(\mathrm{n}=5)$ or rTM pretreatment group $(\mathrm{n}=$ 4) are plotted. The identity of each designated metabolite (a-l) is shown in Supplementary Table. (B) 
Relative abundance of oxidized glutathione, glycodeoxycholate, octanoylcarnitine, and uridine 5'diphosphate (UDP) in the no-anticoagulation group $(n=5)$, rTM pretreatment group $(n=4)$, and rTM treatment at 6-h group $(n=5)$ with reference to samples collected before MRSA inoculation $(n=5)$ are shown. Box plots show the median, lower and upper quartiles, lower and upper extreme values, and outliers. ${ }^{*} \mathrm{P}<0.05 .{ }^{*} \mathrm{P}<0.01$.

\section{Supplementary Files}

This is a list of supplementary files associated with this preprint. Click to download.

- SupplementaryTableMetabolite.docx 